\title{
Potent peptide-conjugated silicon phthalocyanines for tumor photodynamic therapy
}

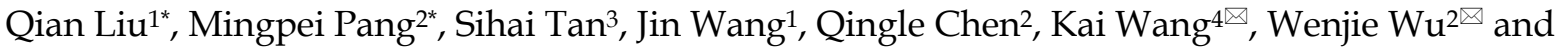 \\ Zhangyong Hong $1{ }^{\bowtie}$ \\ 1. State Key Laboratory of Medicinal Chemical Biology, Tianjin Key Laboratory of Protein Sciences, College of Life Sciences, Nankai University, Tianjin 300071, \\ P. R. China; \\ 2. College of Material Science and Chemical Engineering, Tianjin University of Science and Technology, Tianjin 300457, P. R. China; \\ 3. Tianjin University of Traditional Chinese Medicine, Tianjin 300193, P. R. China; \\ 4. International Medicine Center, Tianjin Hospital, Tianjin 300457, P. R. China. \\ * These authors contributed equally to this work. \\ $\square$ Corresponding authors: Zhangyong Hong, E-mail: hongzy@nankai.edu.cn; Tel.: +86 022 23498707. Wenjie Wu, E-mail: wwjie@tust.edu.cn; Tel.: +86 022 \\ 23502875. Kai Wang, E-mail: wangkai0286@126.com. Tel.: +86 02223003610. \\ (c) Ivyspring International Publisher. This is an open access article distributed under the terms of the Creative Commons Attribution (CC BY-NC) license \\ (https://creativecommons.org/licenses/by-nc/4.0/). See http://ivyspring.com/terms for full terms and conditions.
}

Received: 2017.08.14; Accepted: 2017.10.15; Published: 2018.01.01

\begin{abstract}
Phthalocyanines (Pcs) are a group of promising photosensitizers for use in photodynamic therapy (PDT). However, their extremely low solubility and their strong tendency to aggregate in aqueous solution greatly restrict their application. Conjugation of Pc macrocycles with peptide ligands could be a very useful strategy to optimize the physical properties of Pcs not only by increasing their water solubility and reducing their aggregation but also by endowing the conjugates with a tumor-targeting capability. To develop highly potent photosensitizers for tumor PDT, we prepared new peptide-conjugated photosensitizers using silicon $\mathrm{Pc}(\mathrm{SiPc})$, which has much higher photodynamic activity than zinc Pcs, as the light activation moiety and the cRGDfK peptide (or simply CRGD) as the peptide moiety. A polyethylene glycol linker and an extra carboxylic acid group were also tested for introduction into the conjugates to optimize the conjugate structure. The conjugates' photophysical and photodynamic behaviors were then carefully evaluated and compared using in vitro and in vivo experiments. One of the prepared conjugates, RGD-(Linker) $)_{2}$-Glu-SiPc, showed excellent physical properties and photodynamic activity, with an $\mathrm{EC}_{50}$ (half maximal effective concentration) of 10-20 $\mathrm{nM}$ toward various cancer cells. This conjugate eradicated human glioblastoma U87-MG tumors in a xenograft murine tumor model after only one dose of photodynamic treatment, with no tumor regrowth during observation for up to 35 days. The conjugate RGD-(Linker) ${ }_{2}-\mathrm{Glu}$-SiPc thus showed highly promising potential for use in tumor treatment.
\end{abstract}

Key words: photodynamic therapy, silicon phthalocyanine, integrin, photosensitizer, peptide conjugate.

\section{Introduction}

Photodynamic therapy (PDT), as a highly localized and tumor-specific treatment modality, has emerged as an important therapeutic modality in the management of cancer [1-4]. Phthalocyanines (Pcs) are a group of promising photosensitizers for use in PDT [5-7] due to their advantageous photophysical properties, including intense light absorption (molar extinction coefficient $\varepsilon>1 \times 10^{5} \mathrm{~L} \mathrm{~mol}^{-1} \mathrm{~cm}^{-1}$ ) in the red to near-infrared region $(>650 \mathrm{~nm})$ and high quantum yields of singlet oxygen formation [8]. However, Pc-based photosensitizers have the significant disadvantages of extremely low solubility and a strong tendency to aggregate in aqueous solution due to the hydrophobic nature of the planar Pc macrocycle structure [9-10], which render Pcs photodynamically inactive in aqueous media [11-12]. Modifications are thus necessary for the biological application of Pcs. Conjugation of Pc macrocycles 
with peptide ligands could be a very useful strategy to optimize the physical properties of Pcs [13]. The conjugation of Pcs with hydrophilic peptide ligands can markedly increase the water solubility of the whole conjugate and greatly reduce the aggregation of Pcs [14]. Moreover, certain peptide ligands have a receptor-targeting capability, which is helpful for receptor-mediated internalization of Pcs [15]. These dual functions make peptide conjugation a useful strategy for Pc modification.

In the literature, zinc Pcs are the most used Pcs for the design of peptide-conjugated photosensitizers. For example, van Lier et al. synthesized a series of water-soluble zinc Pc-peptide conjugates targeting gastrin-releasing peptide (GRP) and integrin receptors, and these conjugates were screened for their photodynamic efficacy against various cancer cell lines [16]. Vicente et al. synthesized four zinc Pc-peptide conjugates designed to target the epidermal growth factor receptor (EGFR), and several of them showed efficient targeting capabilities, although their photocytotoxicity toward cancer cells was not very potent [17]. Our group also synthesized conjugates of hydrophilic-modified zinc Pcs with an EGFR-binding peptide [18] or folic acid ligand [19], with the aims of increasing the tumor-targeting capability and reducing background accumulation.

However, zinc Pcs have only very low photodynamic activities, with $\mathrm{EC}_{50}$ (half maximal effective concentration) values of approximately 1-10 $\mu \mathrm{M}$ [20-23]. Photodynamic activity is one most important parameters influencing the treatment outcome of photosensitizers. Therefore, to date, very few peptide-Zinc Pc conjugates have been successfully confirmed as promising photosensitizers for in vivo photodynamic cancer therapy. Compared with zinc Pcs, silicon Pcs (SiPcs) consistently have much higher photodynamic activity; the literature has shown that they always have $\mathrm{EC}_{50}$ values in the nanomolar range [24-27]. For example, PC-4, an asymmetric axially substituted $\mathrm{SiPc}$, has an $\mathrm{EC}_{50}$ in the low nanomolar range [28-29]. Therefore, use of SiPcs to construct peptide conjugates may offer a solution to greatly improving the photodynamic activity of Pc-peptide conjugates, although not much relevant work has been reported to date [4, 30-31]. Here, we endeavored to design and synthesize this type of peptide-conjugated photosensitizer using SiPc as the light activation moiety and a short cyclic sequence, Arg-Gly-Asp-d-Phe-Lys (cRGDfK peptide, or simply cRGD), as the peptide moiety, with the goal of developing highly potent photosensitizers for tumor PDT. The cRGD peptide has a high affinity for the $\alpha_{v} \beta_{3}$ integrin receptor, which is widely expressed on tumor blood vessels, but not in normal tissues [32-34].
Conjugation of the cRGD peptide significantly improved the druggability properties of SiPcs, such as their water solubility, aggregation, and tumortargeting capability.

However, the very strong hydrophobicity and extremely low water solubility of SiPcs may still result in big problems for the design of this type of conjugate. Unmodified SiPcs have extremely low water solubility and thus cannot be dissolved even in dimethylsulfoxide (DMSO). The strong hydrophobicity of SiPcs also greatly affects the affinity of peptide ligands. In the literature, chemical modifications of Pcs through the attachment of hydrophilic substituents, such as sulfonates, carboxylates, quaternized amino groups, carbohydrate or polyhydroxylate, to peripheral positions in the macrocycle are strategies generally used to increase the water solubility of Pcs, and especially zinc Pcs. However, the peripheral modification of SiPcs using hydrophilic groups is very difficult, and to date, no relevant practical methods have been published. To solve the problem of strong hydrophobicity associated with SiPcs, we opted to add a proper length of polyethylene glycol (PEG) linker between the Pc macrocycle and the peptide ligand, with the aims of both increasing the water solubility of the whole conjugate and avoiding the direct connection of these two moieties [35-36]. Moreover, a more strongly hydrophilic carboxylic group was tested for incorporation in the conjugate to further increase the conjugate's water solubility. Here, we report the synthesis and photophysical and biological evaluation of the resultant series of SiPc conjugates. The influence of the PEG linker length and carboxylic acid substitution on the biological behavior of the conjugates and the in vivo tumor treatment potential were carefully evaluated.

\section{Results and discussion}

\section{Molecular design of cRGD-conjugated SiPcs}

We designed the peptide-conjugated Pc photosensitizers by conjugation of an axially substituted SiPc with the cRGD peptide ligand, with the aim of developing new potent tumor-targeting photosensitizers for PDT (Fig. 1 and Scheme 1). The SiPc with axial substitution of the carboxylic group for peptide ligation, which has relatively strong absorption in the near-infrared region (ca. $\lambda=681 \mathrm{~nm}$, $\log \varepsilon=5.23$ ) and a high singlet oxygen quantum yield $(0.32)$, was used as the photosensitizer moiety. The cRGD peptide was used for coupling with the Pc for targeting purposes and increased water solubility. The strong hydrophobic property of the SiPc macrocycle may have a significant influence on the 
receptor affinity of the cRGD ligand; thus, short or long PEG linkers were also introduced into the conjugates to increase the distance between the ligand and the SiPc macrocycle. A glutamic acid residue with a free strongly hydrophilic carboxylic acid group was also added to one conjugate to test if a further increase in hydrophilicity affected the biological function of the conjugates.

\section{Synthesis procedure}

Scheme 1 shows the synthesis route used to prepare the conjugates. The cyclic cRGD peptide ligands with or without the PEG linker and glutamic acid residue (cRGD, cRGD-Linker, cRGD-(Linker) 2 and cRGD-(Linker) ${ }_{2}$-Glu) were prepared using the Fmoc solid-phase peptide synthesis protocol with 2-chlorotrityl chloride resin as the solid support (see the synthetic details in the Supporting Information). The synthesis of SiPc-PQ 2 with amine handles started from the commercially available $\mathrm{SiPcCl}_{2} 1$ with $42 \%$ yield by reaction with 1-piperazineethanol in pyridine. Then SiPc-COOH 3 with carboxylic group handles was obtained with $76 \%$ yield by coupling SiPc-PQ 2 with oxalic anhydride. SiPc-COOH 3 was then coupled with cRGD or PEG-modified cRGD peptides via the typical condensation reaction between the carboxyl and the free amino groups at the $\mathrm{N}$-termini of the cRGD ligands at room temperature to produce conjugates $4,5,6$ and 7 . These conjugates could be obtained in pure form by purification by high-performance liquid chromatography (HPLC) after repeated precipitations with diethyl ether and dissolution in DMSO. The green color of the Pc moiety assisted with the precipitation process. The conjugates were characterized by spectroscopy and analysis of their mass spectra, and their purity was analyzed by HPLC (Fig. S2 in the Supporting Information).

\section{Electronic absorption and photophysical properties}

\section{Absorption spectrum study}

The UV-Vis absorption spectra of conjugates 4,5 , 6 and 7 showed an intense, sharp Q band at $\lambda=680$ $\mathrm{nm}$ in DMSO (Fig. 2 and Table 1), a typically non-aggregated form, which strictly followed the Beer-Lambert law (Fig. S3). DMSO is known to prevent the aggregation of Pcs; however, it is not an appropriate solvent for the evaluation of biological applications. In contrast to the non-conjugated Pcs, all

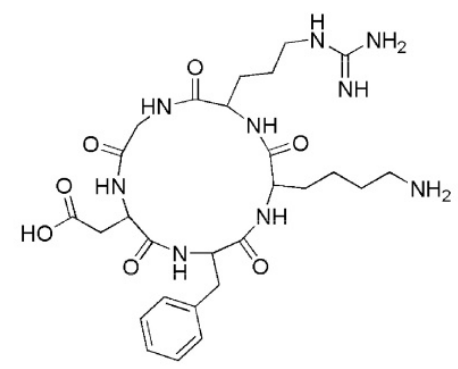

cRGD (cRGDfK peptide)

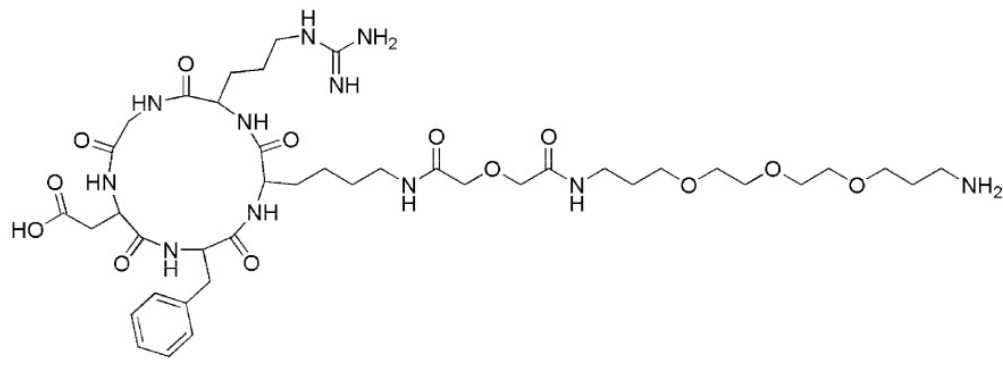

cRGD-Linker
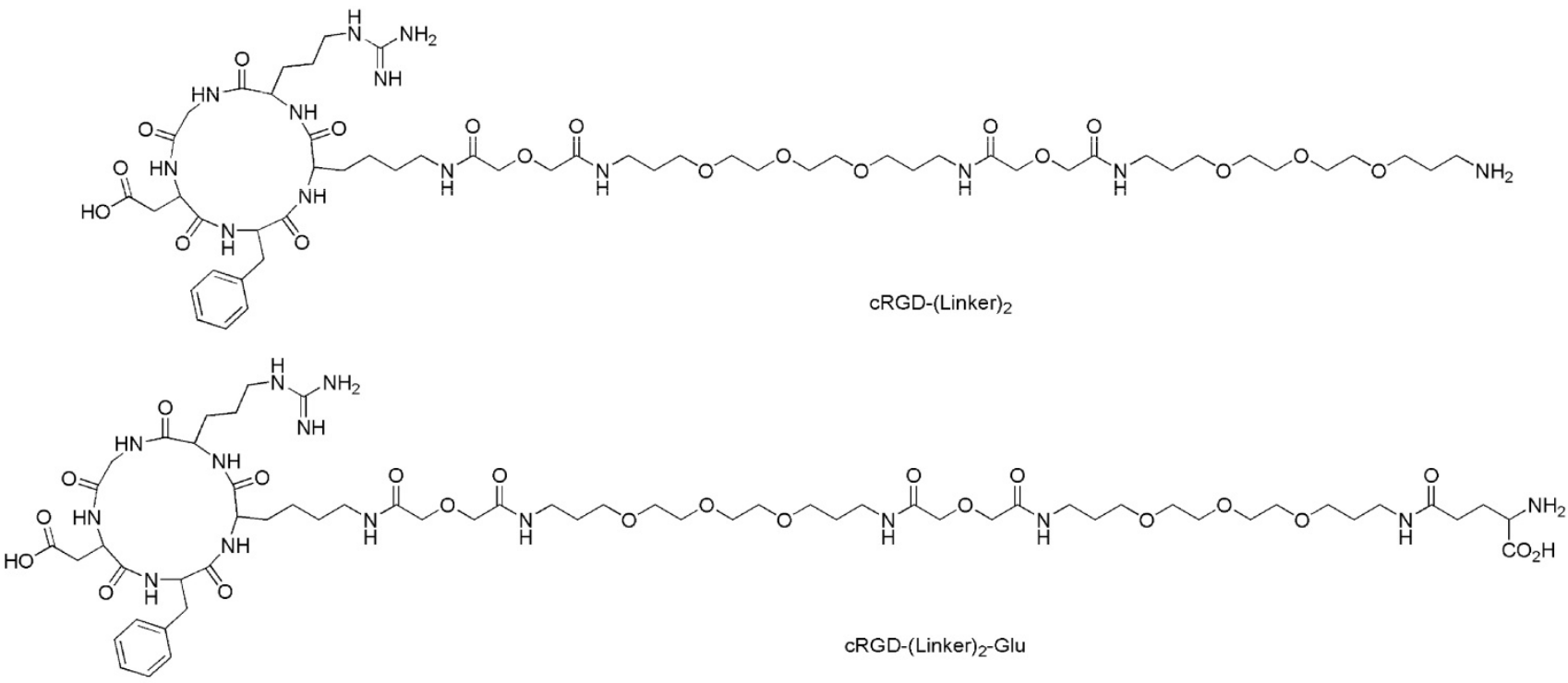

Figure 1. The structures of the cRGD ligands (cRGD, cRGD-Linker, cRGD-(Linker) $)_{2}$ and cRGD-(Linker) ${ }_{2}-\mathrm{Glu}$ ). 
of the conjugates showed good solubility in aqueous solution, and especially conjugate 7 , which had an extra glutamic acid residue. Therefore, the conjugates' photochemical properties were further tested in aqueous solutions, including 10\% Triton X-100 aqueous solution, 1\% Cremophor EL (CEL) aqueous solution $\left(\mathrm{V}_{\mathrm{CEL}} / \mathrm{V}_{\text {water }}=1: 99\right)$ and phosphate-buffered saline (PBS) solution. As shown in Fig. 2, all of these compounds aggregated in PBS solution, as shown by the broadened $Q$ band at $\lambda=600-700 \mathrm{~nm}$. However, the spectra for the conjugates in the $10 \%$ Triton X-100 aqueous solution were similar to those for the conjugates in DMSO, especially for RGD-(Linker) ${ }_{2}$-Glu-SiPc, whose absorption spectrum nearly completely recovered from aggregation. Using a 1\% CEL aqueous solution as the solvent, which is a relatively weak lipid environment, several of these conjugates remained in aggregated forms, characterized by broad $\mathrm{Q}$ bands, whereas RGD-(Linker) ${ }_{2}$-Glu-SiPc showed nearly $100 \%$ recovery of its spectra, as indicated by the same pattern as for the conjugate in DMSO. These results indicate that conjugation with the hydrophilic peptide and modification with the PEG linker and extra carboxylic acid group can significantly influence the water solubility of SiPcs and are very helpful for solving the aggregation problem of SiPcs. Although the conjugates still existed in aggregated forms in PBS, the addition of $10 \%$ Triton and even 1\% CEL significantly disaggregated the peptide-conjugated SiPcs, especially for RGD-(Linker) ${ }_{2}$-Glu-SiPc, which has a longer PEG linker and an extra carboxylic acid group. Adding a glutamic acid residue could obviously increase the solubility of the conjugates, causing the conjugates to be more easily de-aggregated. A lipid aqueous solution could better mimic the physiologic conditions of the cell membrane environment than a pure water solution; this is especially the case for 1\% CEL in aqueous solution, which has a very low lipid concentration. The disaggregation of the photosensitizers under physiologic conditions is very important for their biological application, as only the disaggregated form of photosensitizers has photodynamic activity.

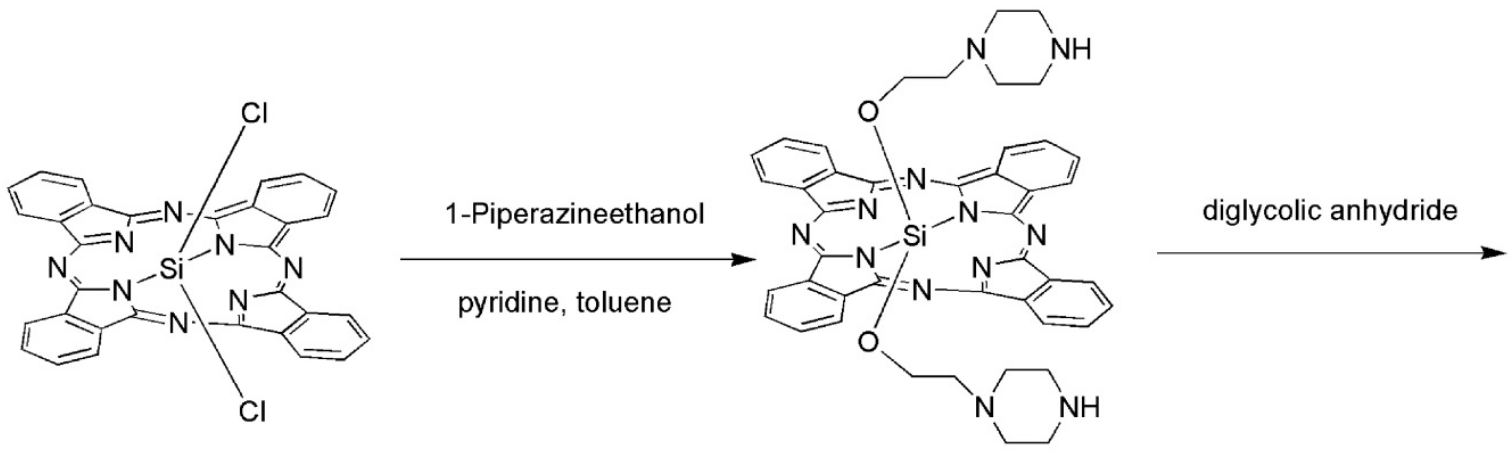

1, $\mathrm{SiPcCl}_{2}$

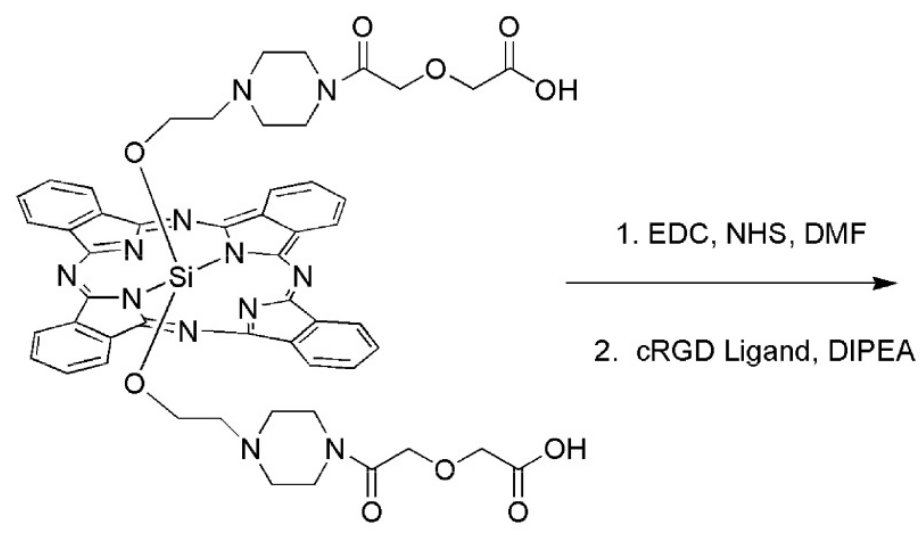

3. $\mathrm{SiPc}-\mathrm{COOH}$
2, SiPc-PQ

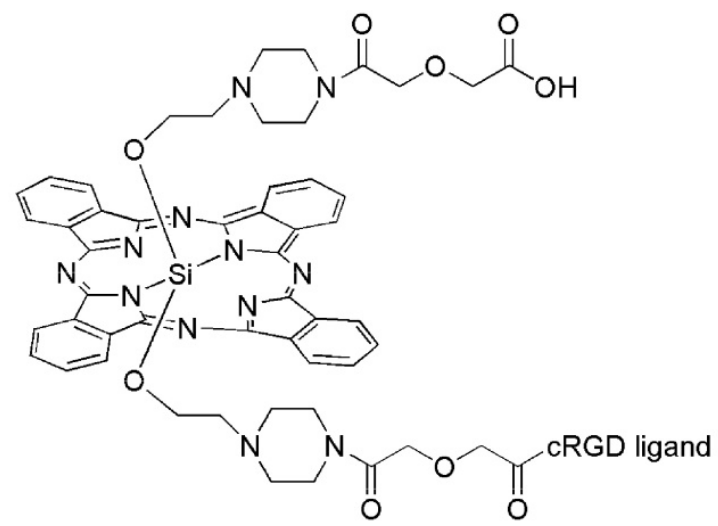

4, RGD-SiPc (ligand = cRGD)

5, RGD-Linker-SiPc (ligand = cRGD-Linker)

6, RGD-(Linker) $)_{2}-\mathrm{SiPc}$ (ligand $\left.=\mathrm{cRGD}-(\text { Linker })_{2}\right)$

7, RGD-(Linker) ${ }_{2}$-Glu-SiPc (ligand $=$ cRGD-(Linker) $)_{2}$-Glu)

Scheme 1. Synthesis route for the axially substituted SiPc-PQ 2; SiPc-COOH 3; and the peptide conjugates RGD-SiPc 4, RGD-Linker-SiPc 5, RGD-(Linker)2-SiPc 6, and RGD-(Linker) $)_{2}$-Glu-SiPc 7. 
A

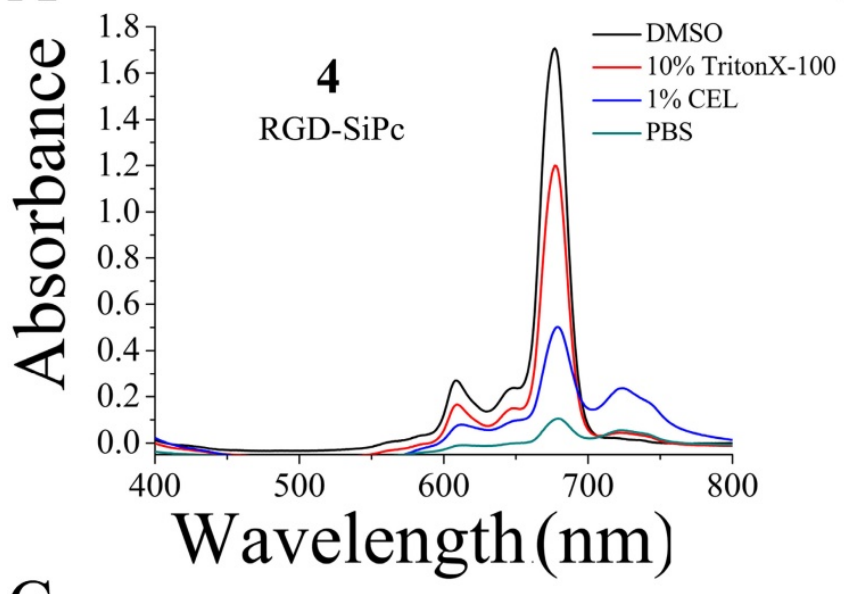

C

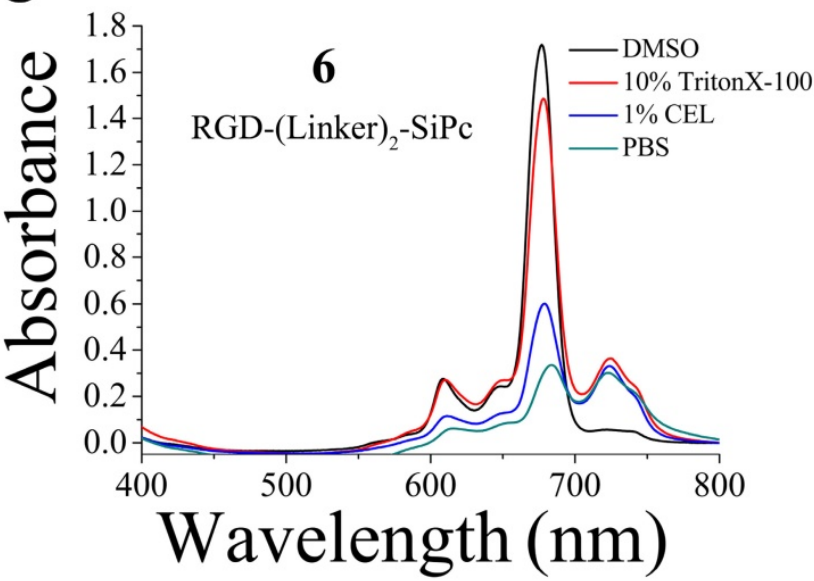

B

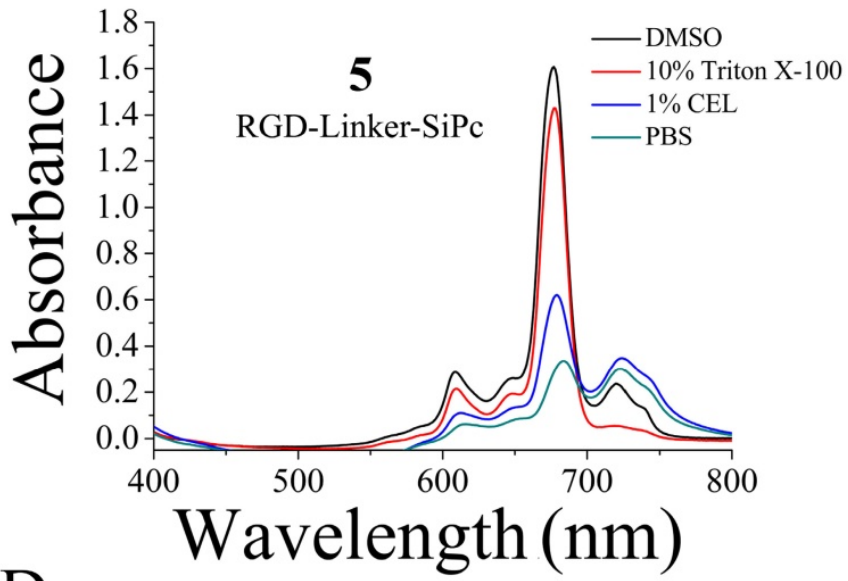

$\mathrm{D}$

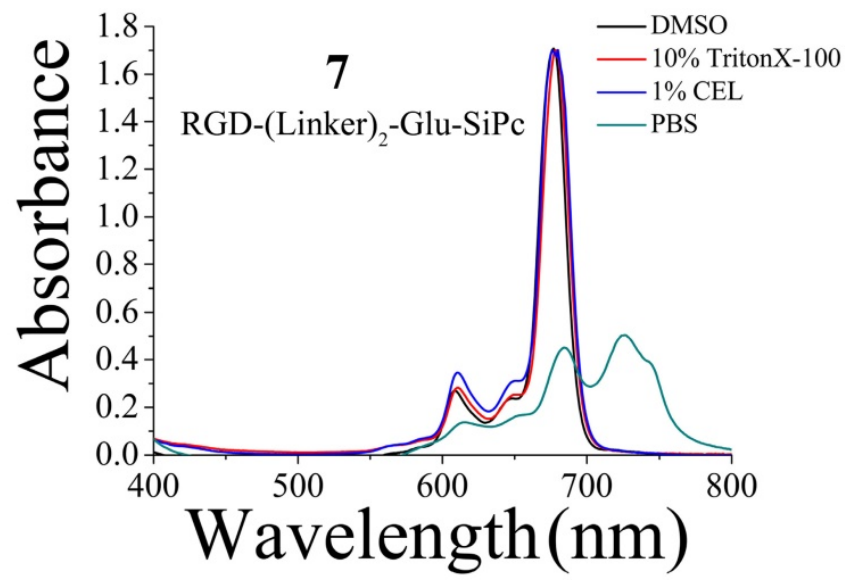

Figure 2. UV-Vis absorption spectra of the conjugates RGD-SiPc 4 (A), RGD-Linker-SiPc 5 (B), RGD-(Linker)2-SiPc 6 (C) and RGD-(Linker) 2 -Glu-SiPc 7 (D) in different solvents (i.e., DMSO, 10\% Triton X-100 aqueous solution, 1\% CEL aqueous solution and PBS solution). The concentration of the compounds for absorption determination was $10 \mu \mathrm{M}$.

\section{Fluorescence quantum yields, lifetimes and singlet oxygen quantum yield}

The fluorescence data and singlet oxygen quantum yields of the conjugates were determined in DMSO as described previously [18, 19]. As shown in Table 2, the cRGD moiety did not exert a significant influence on the electronic absorption and photophysical properties of the Pc core. The conjugates had similar maximum emission and excitation wavelengths with non-conjugated SiPc-PQ 2 and $\mathrm{SiPc}-\mathrm{COOH}$ 3. Their fluorescence quantum yields are also similar, but they showed a slightly reduced fluorescence decay time compared with SiPc-PQ 2 and SiPc-COOH 3. All of the conjugates also had relatively high singlet oxygen quantum yields, but interestingly, conjugation with the peptide and linkers seemed to slightly increase the singlet oxygen quantum yields of the conjugates. RGD-(Linker) ${ }_{2}$-Glu-SiPc 7 had the highest yield (0.39). Singlet oxygen $\left({ }^{1} \mathrm{O}_{2}\right)$ is believed to be the major cytotoxic agent involved in PDT, and an increase in the singlet oxygen quantum yield should be beneficial for PDT.

\section{In vitro photodynamic activity}

The in vitro photodynamic activity of the conjugates was evaluated against tumor cell lines by MTT assay. Human glioblastoma U87-MG cells, human prostate carcinoma 22RV1 and PC 3 cells, and human epidermoid carcinoma A431 cells were used for the evaluation. U87-MG, 22RV1 and PC3 cells highly express the $\alpha_{v} \beta_{3}$ integrin receptor $\left(\alpha_{v} \beta_{3}{ }^{+}\right)$, and A431 cells show low levels of expression of the $\alpha_{v} \beta_{3}$ integrin receptor $\left(\alpha_{v} \beta_{3^{-}}\right)$. All of the cells were incubated with the conjugates, which were dissolved in aqueous culture media in the presence of $1.0 \% \mathrm{CEL}$, for $4 \mathrm{~h}$ before light illumination at a dosage of 40 $\mathrm{mW} / \mathrm{cm}^{2}$ for $15 \mathrm{~min}\left(36 \mathrm{~J} / \mathrm{cm}^{2}\right)$ at $670 \mathrm{~nm}$. Fig. 3 and Fig. 4 show the corresponding dose-dependent survival curves without or with laser illumination, and Table 3 shows the corresponding $\mathrm{EC}_{50}$ values of the conjugates toward the cells. None of the four conjugates showed significant cytotoxicity in the dark 
toward the above cell lines at concentrations up to 0.5 $\mu \mathrm{M}$ (to avoid solubility problems, no higher concentrations were tested), and light irradiation alone did not affect cell viability (Fig. 3). However, upon illumination with light, cell viability was strongly reduced in the presence of very low concentrations of the conjugates. All of the conjugates had $\mathrm{EC}_{50}$ values in the nanomolar range, and the conjugate RGD-(Linker) ${ }_{2}$-Glu-SiPc 7 had the most potent photodynamic activity, with $\mathrm{EC}_{50}$ values of $17.1 \mathrm{nM}, 16.7 \mathrm{nM}, 16.2 \mathrm{nM}$ and $50.4 \mathrm{nM}$ toward U87-MG, 22RV1, PC3 and A431 cells, respectively (Fig. 4 and Table 3). The carboxylic group of the glutamic acid residue seems to have played a significant role in the improvement of the PDT activity of the conjugates.

Table 1. Absorption spectral data for SiPc-PQ 2, SiPc-COOH 3 , RGD-SiPc 4, RGD-Linker-SiPc 5, RGD-(Linker)2-SiPc 6 and RGD-(Linker)2-Glu-SiPc 7 in DMSO, 10\% Triton X-100 aqueous solution, $1 \%$ CEL aqueous solution and PBS solution.

\begin{tabular}{|c|c|c|}
\hline Compound & Solvent & $\lambda_{\max } / \mathrm{nm}(\log \varepsilon)$ \\
\hline \multirow{4}{*}{$\begin{array}{c}2 \\
\text { SiPc-PQ }\end{array}$} & DMSO & $676(5.23)$ \\
\hline & $10 \%$ Triton & $678(4.85)$ \\
\hline & $1 \%$ CEL & $676(4.07)$ \\
\hline & PBS & $682(3.38)$ \\
\hline \multirow{4}{*}{$\begin{array}{c}\mathbf{3} \\
\text { SiPc-COOH }\end{array}$} & DMSO & $681(5.23)$ \\
\hline & $10 \%$ Triton & $674(4.98)$ \\
\hline & $1 \%$ CEL & $678(4.07)$ \\
\hline & PBS & $683(3.59)$ \\
\hline \multirow{4}{*}{$\begin{array}{c}\mathbf{4} \\
\text { RGD-SiPc }\end{array}$} & DMSO & $678(5.22)$ \\
\hline & $10 \%$ Triton & $679(5.07)$ \\
\hline & $1 \% \mathrm{CEL}$ & $679(4.70)$ \\
\hline & PBS & $680(4.02)$ \\
\hline \multirow{4}{*}{$\begin{array}{c}\mathbf{5} \\
\text { RGD-Linker-SiPc }\end{array}$} & DMSO & $677(5.20)$ \\
\hline & $10 \%$ Triton & $679(5.17)$ \\
\hline & $1 \%$ CEL & 679 (4.79) \\
\hline & PBS & $684(4.53)$ \\
\hline \multirow{5}{*}{$\begin{array}{c}\mathbf{6} \\
\text { RGD-(Linker) })_{2} \text {-SiPc }\end{array}$} & DMSO & $677(5.24)$ \\
\hline & $10 \%$ Triton & $678(5.17)$ \\
\hline & $1 \% \mathrm{CEL}$ & $679(4.78)$ \\
\hline & PBS & $684(4.53)$ \\
\hline & DMSO & $677(5.23)$ \\
\hline \multirow{3}{*}{$\begin{array}{c}7 \\
\text { RGD-(Linker) })_{2} \text {-Glu-SiPc }\end{array}$} & $10 \%$ Triton & $679(5.22)$ \\
\hline & $1 \%$ CEL & $676(5.23)$ \\
\hline & PBS & $684(4.65)$ \\
\hline
\end{tabular}

The difference in the photocytotoxicities of the conjugates toward receptor-positive and receptor-negative cells was not significant. Similar results were observed for the recently reported cRGD-conjugated pyropheophorbide a and zinc Pc, which exhibited similar photocytotoxicities toward the $\alpha_{v} \beta_{3}{ }^{+}$U87-MG cells and $\alpha_{v} \beta_{3}{ }^{-}$A431 or MCF-7 cells after drug incubation [34, 37]. The uptake of these conjugates may not be solely due to the number of receptors on the cell surface; another route, such as through the direct anchoring of these conjugates to the cell surface membrane due to the strong hydrophobicity of the SiPc moiety, may exist. In the future, strategies to reduce the strong hydrophobicity of the SiPc moiety may be required to increase the cell selectivity of the conjugates.

\section{In vivo PDT}

RGD-(Linker) ${ }_{2}$-Glu-SiPc $\quad 7$ is a better photosensitizer than the other three conjugates for tumor PDT. This conjugate had the highest PDT activity in the cell-based activity assay, had better water solubility, and was more easily disaggregated in aqueous solution. Therefore, this conjugate was selected for the animal treatment experiment. We specifically used U87-MG murine xenograft model to study the photodynamic effect of this conjugate. In this model, U87-MG cells were injected subcutaneously into the right flanks of BALB/c nude mice. When the tumor volume reached $100 \mathrm{~mm}^{3}$, the conjugate RGD-(Linker) ${ }_{2}-\mathrm{Glu}-\mathrm{SiPc} 7$ or saline, as the control, was injected intravenously via the caudal vein, followed by light illumination $\left(200 \mathrm{~mW} / \mathrm{cm}^{2}\right.$ for $16 \mathrm{~min}$ ). As shown in Fig. 5, in the control group, which received the saline injection followed by light illumination, the treatment had no effect on reducing the tumor growth rate, and the tumor sizes were found to increase dramatically, up to $1500 \mathrm{~mm}^{2}$, in less than 25 days. In contrast, the tumor sizes in the conjugate treatment groups, which received both the conjugate injection and light illumination, were greatly attenuated. All tumors were irradiated at approximately day 14, and no tumors grew again during observation for up to 35 days. During the whole treatment, no detectable body weight loss was observed, indicating that neither the irradiation nor the conjugate RGD-(Linker) ${ }_{2}-$ Glu-SiPc 7 caused serious side effects in the mice. The extremely high efficacy of the PDT based on the conjugate RGD-(Linker) ${ }_{2}$-Glu-SiPc 7 in the U87-MG tumors demonstrated that this conjugate has high clinical potential for cancer therapy.

\section{Conclusion}

Herein, we designed and synthesized peptide-conjugated SiPcs by conjugation of an axially substituted SiPc with the cRGD peptide ligand, with the aim of developing new potent photosensitizers for tumor PDT. Conjugation with the cRGD peptide greatly increased the water solubility of the SiPc. After introducing two PEG linkers and one strong hydrophilic carboxylic acid group to further increase the hydrophilicity, the prepared conjugate 
RGD-(Linker) 2 -Glu-SiPc $\quad 7 \quad$ showed excellent properties and photodynamic activity, with $\mathrm{EC}_{50}$ values of 10-20 nM toward various cancer cells. This conjugate eradicated the U87-MG tumors in the xenograft murine tumor model after only one dose of photodynamic treatment, and no tumors grew again during observation for up to 35 days. These results demonstrate that PDT based on the conjugate RGD-(Linker) ${ }_{2}$-Glu-SiPc $\mathbf{7}$ has high potential for use in tumor treatments.

Table 2. Photophysical and photochemical parameters for SiPc-PQ 2, SiPc-COOH 3, RGD-SiPc 4, RGD-Linker-SiPc 5, RGD-(Linker) ${ }_{2}$-SiPc 6 and RGD-(Linker) ${ }_{2}$-Glu-SiPc 7 in DMSO. $\Phi_{\mathrm{F}}$ : fluorescence quantum yield; TF: fluorescence lifetime; $\Phi_{\Delta}$ : singlet oxygen quantum yield. For the reference standard, $\mathrm{ZnPc}$ was used; $\Phi_{\Delta}=0.67$.

\begin{tabular}{ccccccc}
\hline Compound & $\lambda_{\text {abs }}[\mathrm{nm}](\log \varepsilon)$ & $\lambda_{\text {ex }}[\mathrm{nm}]$ & $\lambda_{\text {em }}[\mathrm{nm}]$ & $\Phi_{\mathrm{F}}$ & $\tau_{\mathrm{F}[\mathrm{ns}]}$ & $\Phi_{\Delta}$ \\
\hline SiPc-PQ, 2 & $676(5.23)$ & 671 & 678 & 0.08 & 5.04 & 0.26 \\
SiPc-COOH, 3 & $681(5.22)$ & 676 & 682 & 0.09 & 4.48 & 0.32 \\
RGD-SiPc, $\mathbf{4}$ & $678(5.22)$ & 675 & 682 & 0.08 & 4.60 & 0.34 \\
RGD-Linker-SiPc, $\mathbf{5}$ & $677(5.20)$ & 674 & 681 & 0.10 & 4.38 & 0.34 \\
RGD-(Linker)2-SiPc, $\mathbf{6}$ & $677(5.24)$ & 676 & 683 & 0.12 & 3.06 & 0.38 \\
RGD-(Linker)2-Glu-SiPc, $\mathbf{7}$ & $677(5.23)$ & 677 & 684 & 0.12 & 3.01 & 0.39 \\
\hline
\end{tabular}

Table 3. $\mathrm{EC}_{50}$ values of SiPc-COOH 3, RGD-SiPc 4, RGD-Linker-SiPc 5, RGD-(Linker)2-SiPc 6 and RGD-(Linker) $)_{2}-$ Glu-SiPc 7 toward U87-MG, 22RV1, PC3 and A431 cells. Data acquisition was performed using GraphPad Prism software.

\begin{tabular}{ccccc}
\hline $\mathrm{EC}_{50}(\mathrm{nM})$ & $\mathrm{U} 87-\mathrm{MG}$ & $2^{22 \mathrm{RV} 1}$ & PC3 & A431 \\
\hline SiPc-COOH, 3 & 39.9 & 30.7 & 37.1 & 40.1 \\
RGD-SiPc, $\mathbf{4}$ & 132.3 & 67.9 & 79.3 & 160.0 \\
RGD-inker-SiPc, $\mathbf{5}$ & 261.7 & 292.3 & 229.3 & 461.5 \\
RGD-(Linker) 2 -SiPc, $\mathbf{6}$ & 250.6 & 266.9 & 310.4 & 624.9 \\
RGD-(Linker) 2 -Glu-SiPc, $\mathbf{7}$ & 17.3 & 16.7 & 15.5 & 50.6 \\
\hline
\end{tabular}

A

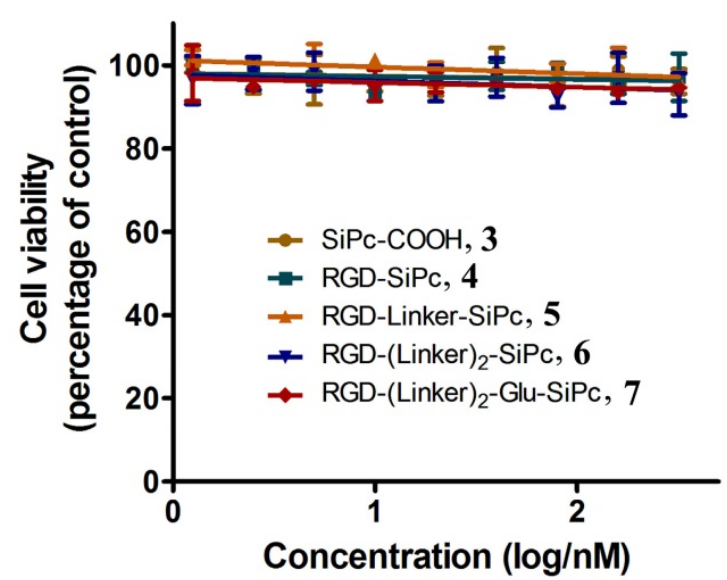

B

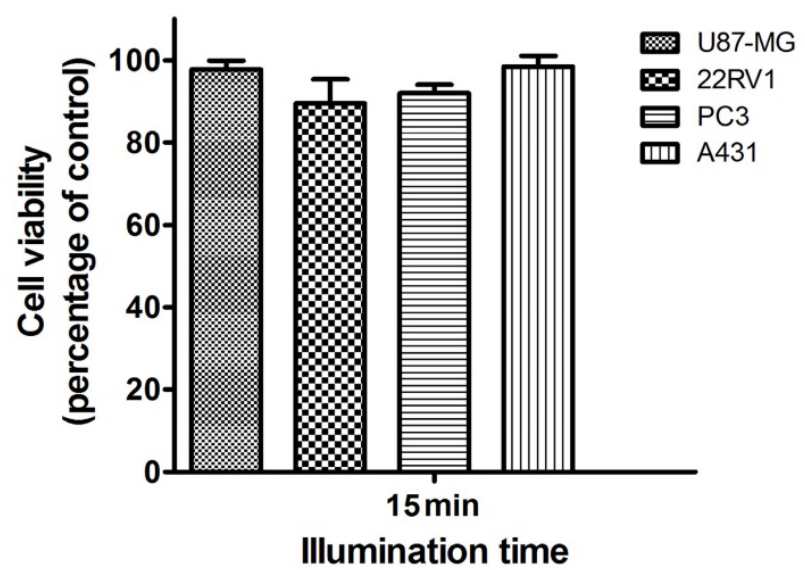

Figure 3. Cytotoxicity of the conjugates in the dark and cytotoxicity of the light alone. The viability of U87-MG cells (A) incubated with SiPc-COOH 3, RGD-SiPc 4, RGD-Linker-SiPc 5, RGD-(Linker)2-SiPc 6 and RGD-(Linker)2-Glu-SiPc 7 without light and the viability of U87-MG, 22RV1, PC3 and A431 cells with light illumination alone $\left(36 \mathrm{~J} / \mathrm{cm}^{2}\right)(B)$ were determined by MTT assay. 
A

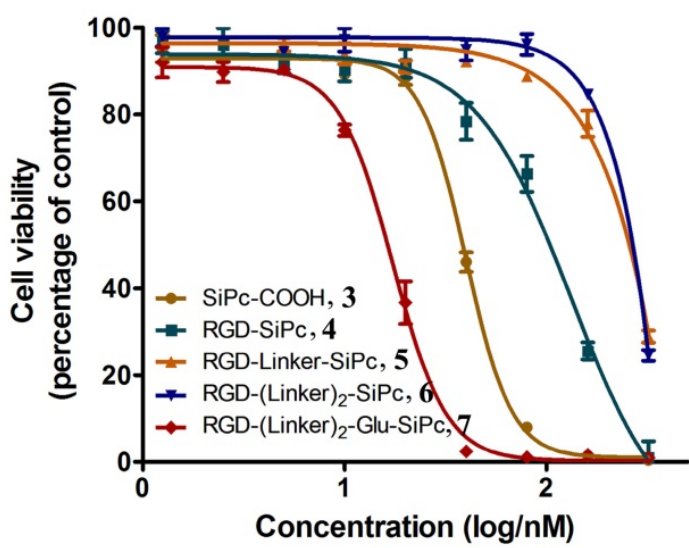

C

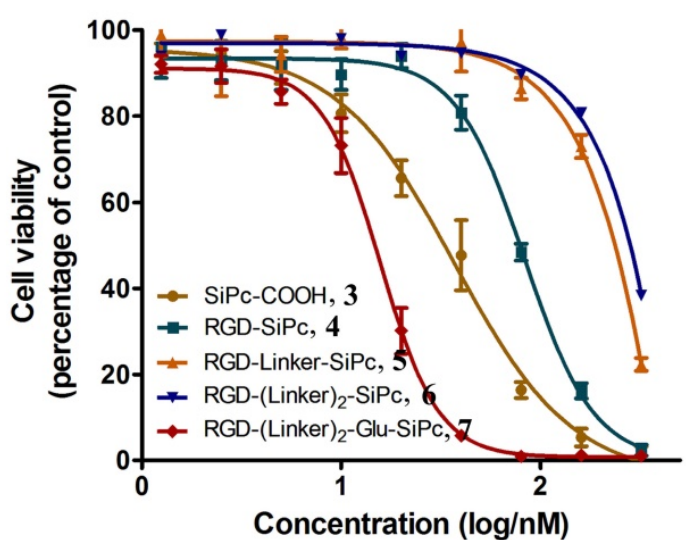

B 22RV1

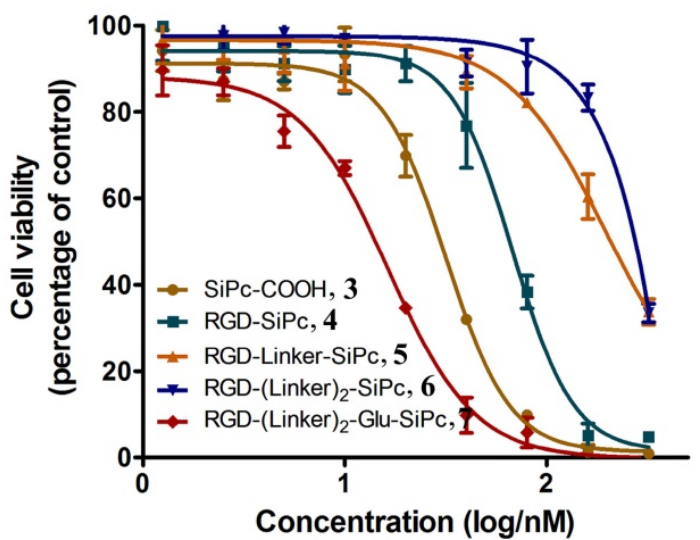

D

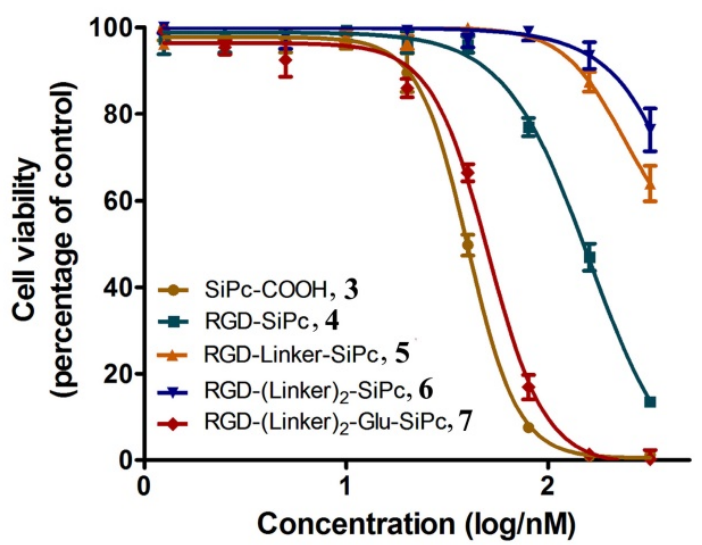

Figure 4. In vitro concentration-dependent photocytotoxicity of SiPc-COOH 3 (circles, dark yellow), RGD-SiPc 4 (squares, olive), RGD-Linker-SiPc 5 (upward triangles, orange), RGD-(Linker)2-SiPc 6 (downward triangles, blue) and RGD-(Linker)2-Glu-SiPc 7 (diamonds, red) on receptor-positive U87-MG (A), 22RV1 (B), and PC3 (C) cells and receptor-negative A431 cells $(D)(n=3$, mean \pm SEM), as determined by MTT assay. The cells were treated with various concentrations of the conjugates for $4 \mathrm{~h}$ before illumination with light $\left(40 \mathrm{~mW} / \mathrm{cm}^{2}, 15 \mathrm{~min}\right)$, and cell viability was determined by MTT assay.

A

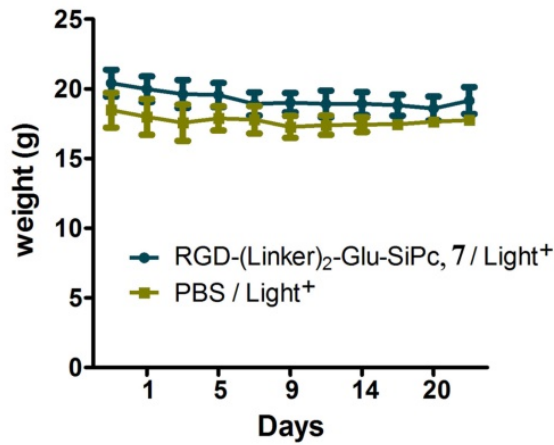

C

\section{PBS / Light ${ }^{+}$ \\ RGD-(Linker) $)_{2}^{-}$ Glu-SiPc, 7 / Light $^{+}$}

B

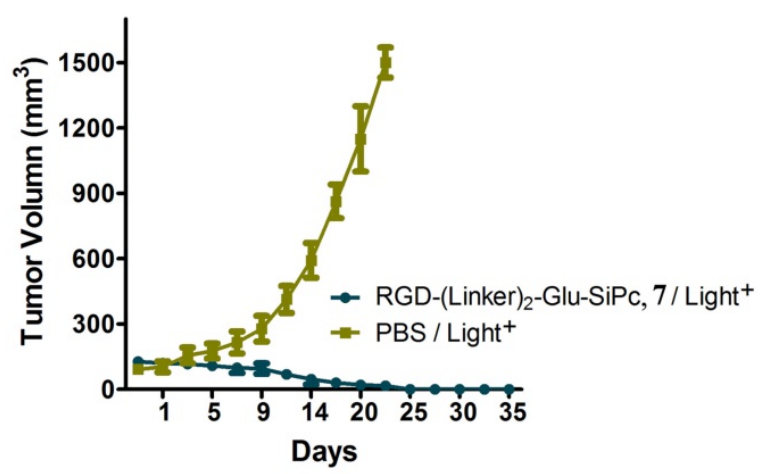

7D

14D

18D

35D

Figure 5. In vivo phototherapeutic activity of the conjugate RGD-(Linker) ${ }_{2}$-Glu-SiPc 7. The body weight (A) and tumor volume (B) changes of the BALB/c nude mice were monitored every other day. Photographs $(C)$ were taken to observe the more visible tumor changes (one representative mouse from each group is shown). All of the mice were irradiated once with $670-\mathrm{nm}$ light for $16 \mathrm{~min}\left(288 \mathrm{~J} / \mathrm{cm}^{2}\right) 4 \mathrm{~h}$ after injection with PBS or RGD-(Linker)2-Glu-SiPc 7 (50 nmol per mouse). 


\section{Experimental section}

\section{Chemicals and materials}

Silicon Pc dichloride $\left(\mathrm{SiPcCl}_{2}, \mathbf{1}\right)$, 1,13-diamino4,7,10-trioxatridecane, 1-ethyl-3-(3dimethyllaminopropyl) carbodiimide hydrochloride $(\mathrm{EDC} \cdot \mathrm{HCl})$ and piperazine ethanol were purchased from Sigma-Aldrich (St. Louis, MO, USA). N-hydroxysuccinimide (NHS), diglycolic anhydride and diisopropylethylamine (DIPEA) were purchased from Heowns (Tianjin, China). Fetal bovine serum (FBS) and antibiotics (penicillin/streptomycin) were obtained from HyClone (Logan, UT, USA). Other chemical reagents were obtained from J\&K Chemical Ltd. (Beijing, China).

\section{Cells and animals}

U87-MG, 22RV1, PC3 and A431 cells were obtained from the Cell Bank of the Chinese Academy of Sciences and cultured in DMEM supplemented with $10 \% \quad(v / v) \quad$ FBS and $1 \% \quad(v / v)$ penicillin/streptomycin at $37{ }^{\circ} \mathrm{C}$ in a $5 \% \mathrm{CO}_{2}$ environment. Cells were typically passaged every day at a 1:2 ratio following harvest with $0.25 \%$ trypsin-EDTA (1X) and phenol red. Female BALB/c nude mice (6 weeks, 18-22 g) were provided by Beijing Vital River Laboratory Animal Technology Co., Ltd (Beijing, China). All of the mice were treated according to the guidelines of the Committee on Animals of Nankai University.

\section{Instruments}

${ }^{1} \mathrm{H}$ and ${ }^{13} \mathrm{C}$ NMR spectra were recorded in DMSO-d6 solutions on a Bruker AV400 $400 \mathrm{MHz}$ spectrometer. Mass spectra were recorded on Varian 7.0T FTMS. UV-Vis spectra were recorded with a USA Cary 5000 spectrophotometer (Varian Co., Palo Alto, USA) using a $1-\mathrm{cm}$ path-length cuvette at room temperature. Fluorescence measurements were performed on a PTI QM/TM/NIR system (Photon Technology International, Birmingham, NJ, USA) equipped with a quartz cell $(1 \mathrm{~cm} \times 1 \mathrm{~cm})$. HPLC (Shimadzu, Kyoto, Japan) was used for purification and analysis.

\section{Synthesis experiments}

\section{Synthesis of SiPc-PQ 2}

1-Piperazineethanol $(20.8 \mathrm{mg}, 0.016 \mathrm{mmol})$ and $\mathrm{SiPcCl}_{2} 1$ (10.0 mg, $\left.0.016 \mathrm{mmol}\right)$ were added to a mixed solution of toluene and pyridine $(1 \mathrm{~mL}, \mathrm{v} / \mathrm{v}=$ 5:1) and refluxed for approximately $10 \mathrm{~h}$. The solvent was then removed under reduced pressure. The residue was diluted with $\mathrm{CH}_{2} \mathrm{Cl}_{2}$ and washed with saline three times, and the combined organic layer was dried over $\mathrm{Na}_{2} \mathrm{SO}_{4}$. The product was concentrated and purified by silica gel chromatography eluted with $\mathrm{MeOH}, \mathrm{CH}_{2} \mathrm{Cl}_{2}$ and a small amount of TEA to yield SiPc-PQ 2 (5.0 mg, 42\%). HRMS (ESI): $\mathrm{m} / \mathrm{z}$ calculated for $\mathrm{C}_{44} \mathrm{H}_{42} \mathrm{~N}_{12} \mathrm{O}_{2} \mathrm{Si}[\mathrm{M}+$ $\mathrm{H}]^{+}$799.3396, found 799.3392 .

\section{Synthesis of SiPc-COOH 3}

SiPc-PQ $2(10.0 \mathrm{mg}, 0.013 \mathrm{mmol})$ and oxydiacetic anhydride $(9.0 \mathrm{mg}, 0.078 \mathrm{mmol})$ were added to $200 \mu \mathrm{L}$ anhydrous DMF and stirred at room temperature for $2 \mathrm{~h}$. The mixture was precipitated and washed with anhydrous diethyl ether to yield SiPc-COOH 3 (10.0 $\mathrm{mg}, \quad 76 \%$ ). HRMS (ESI): $\mathrm{m} / \mathrm{z}$ calculated for $\mathrm{C}_{52} \mathrm{H}_{51} \mathrm{~N}_{12} \mathrm{O}_{10} \mathrm{Si}[\mathrm{M}+\mathrm{H}]^{+}$1031.3615, found 1031.3613.

\section{Synthesis of RGD-SiPc 4}

SiPc-COOH 3 (10.0 mg, $0.010 \mathrm{mmol})$, EDC (1.8 $\mathrm{mg}, 0.009 \mathrm{mmol})$ and NHS (1.1 mg, $0.010 \mathrm{mmol})$ were added to $1.0 \mathrm{~mL}$ anhydrous DMF and stirred at room temperature for $4 \mathrm{~h}$. DIPEA $(2.5 \mathrm{mg}, 0.019 \mathrm{mmol})$ and cRGD $(1.7 \mathrm{mg}, 0.003 \mathrm{mmol})$ were then added to the mixture and stirred at room temperature overnight. The final mixture was precipitated and washed with ethyl ether and dichloromethane three times, followed by HPLC purification to yield RGD-Linker-SiPc 4 (3.8 mg, 78\%). HRMS (ESI): m/z calculated for $\mathrm{C}_{79} \mathrm{H}_{90} \mathrm{~N}_{21} \mathrm{O}_{16} \mathrm{Si}[\mathrm{M}+\mathrm{H}]^{+}$1616.6638, found 1616.6705 .

\section{Synthesis of RGD-Linker-SiPc 5}

SiPc-COOH 3 (10.0 mg, $0.010 \mathrm{mmol})$, EDC (1.8 $\mathrm{mg}, 0.009 \mathrm{mmol})$, and NHS (1.1 mg, $0.010 \mathrm{mmol})$ were added to $200 \mu \mathrm{L}$ anhydrous DMF and stirred at room temperature for $4 \mathrm{~h}$. DIPEA $(2.5 \mathrm{mg}, 0.019 \mathrm{mmol})$ and cRGD-Linker $(2.6 \mathrm{mg}, 0.003 \mathrm{mmol})$ were then added to the mixture and stirred at room temperature overnight. The final mixture was precipitated and washed with ethyl ether and dichloromethane three times, followed by HPLC purification to yield RGD-Linker-SiPc 5 (3.3 mg, 57\%). HRMS (ESI): m/z calculated for $\mathrm{C}_{93} \mathrm{H}_{116} \mathrm{~N}_{23} \mathrm{O}_{22} \mathrm{Si}[\mathrm{M}+\mathrm{H}]^{+}$1934.8429, found 1934.8512 .

\section{Synthesis of RGD-(Linker) ${ }_{2}$-SiPc 6}

SiPc-COOH 3 (10.0 mg, $0.010 \mathrm{mmol})$, EDC (1.8 $\mathrm{mg}, 0.009 \mathrm{mmol})$, and NHS $(1.1 \mathrm{mg}, 0.010 \mathrm{mmol})$ were added to $200 \mu \mathrm{L}$ anhydrous DMF and stirred at room temperature for $4 \mathrm{~h}$. DIPEA $(2.5 \mathrm{mg}, 0.019 \mathrm{mmol})$ and cRGD-(Linker) 2 ( $3.6 \mathrm{mg}, 0.003 \mathrm{mmol})$ were then added to the mixture and stirred at room temperature overnight. The final mixture was precipitated and washed with ethyl ether and dichloromethane three times, followed by HPLC purification to yield RGD-(Linker) $)_{2}$-SiPc 6 (2.6 mg, 40\%). 


\section{Synthesis of RGD-(Linker) ${ }_{2}-$ Glu-SiPc 7}

SiPc-COOH 3 (10.0 mg, $0.010 \mathrm{mmol})$, EDC (1.8 $\mathrm{mg}, 0.009 \mathrm{mmol})$, and NHS (1.1 mg, $0.010 \mathrm{mmol})$ were added to $200 \mu \mathrm{L}$ anhydrous DMF and stirred at room temperature for $4 \mathrm{~h}$. DIPEA $(2.5 \mathrm{mg}, 0.019 \mathrm{mmol})$ and cRGD-(Linker) ${ }_{2}$-Glu $(4.0 \mathrm{mg}, 0.003 \mathrm{mmol})$ were then added to the mixture and stirred at room temperature overnight. The final mixture was precipitated and washed with ethyl ether and dichloromethane three times, followed by HPLC purification to yield RGD-(Linker) ${ }_{2}$-Glu-SiPc 7 (2.5 mg, 32\%).

\section{Photophysical and photochemical properties}

\section{UV-Vis spectra}

The UV-Vis spectra were measured using a USA Cary 5000 with a 1-cm path-length cuvette. The concentration of Pcs was $10 \mu \mathrm{M}$ in the solvents, including DMSO, 10\% Triton X-100 aqueous solution, 1\% CEL aqueous solution and PBS solution. Baselines and spectra were recorded at room temperature in the wavelength range from 500-900 nm, with a 1-nm resolution and a $600 \mathrm{~nm} / \mathrm{min}$ scan rate.

\section{Fluorescence spectra}

The fluorescence emission spectra were recorded in the wavelength range of 600-900 $\mathrm{nm}$ upon excitation at $680 \mathrm{~nm}$, and the fluorescence excitation spectra were recorded in the wavelength range of 500-900 nm upon emission at $695 \mathrm{~nm}$. The concentration of Pcs was $2.0 \mu \mathrm{M}$ in DMSO. The slit widths were 1 and $2 \mathrm{~nm}$ for excitation and emission, respectively.

The natural radiative lifetimes, absolute fluorescence quantum yields and singlet oxygen quantum yields of these compounds were all determined according to our published procedures $[18,19]$. Briefly, the fluorescence decay curves were measured on a PTI QM-4m system (Photon Technology International, Birmingham, NJ, USA) with Felix32 software; the absolute fluorescence quantum yields at room temperature were determined on a PTI QM/TM/NIR system (Photon Technology International, Birmingham, NJ, USA) with an integration sphere attachment (Labsphere, North Sutton, NH, USA); the singlet oxygen quantum yields were determined via a steady-state method using DPBF (1,3-diphenylisobenzofuran, $20 \mu \mathrm{M})$ as the scavenger in DMSO $(10 \mu \mathrm{M}$ of the conjugates) with $\mathrm{ZnPc}\left(\Phi_{\Delta}=0.67\right.$ in DMSO$)$ as the standard.

\section{In vitro photodynamic activity}

In vitro PDT was performed on U87-MG, 22RV1, PC3 and A431 cells. The cells were seeded in 96-well plates $\left(1 \times 10^{6}\right.$ cells per well $)$ and cultured for $24 \mathrm{~h}$ prior to PDT. The cells were then incubated with various concentrations of conjugate $4,5,6$ or 7 in complete DMEM for $4 \mathrm{~h}$, followed by light illumination $\left(670 \mathrm{~nm}, 40 \mathrm{~mW} / \mathrm{cm}^{2}, 15 \mathrm{~min}\right)$. Four hours later, the cell monolayers were incubated with $500 \mu \mathrm{g} / \mathrm{mL}$ MTT solution at $37^{\circ} \mathrm{C}$ for $4 \mathrm{~h}$. Next, the formazan crystals that formed were dissolved in DMSO, and the absorbance of the dissolved formazan crystals at $490 \mathrm{~nm}$ was measured using a 96-well plate reader (BioTek microplate reader). Meanwhile, we carried out experiments to assess the toxicity of the conjugates toward the cell lines in the dark. The cell viability was determined as described above but without light illumination after incubation of the cells with various concentrations of the conjugates. Each experiment was repeated three times with three replicates at each concentration of the conjugate and for each cell line. The cell phototoxicity curves were plotted as a function of the photosensitizer dose, and the $\mathrm{EC}_{50}$ values were calculated.

\section{In vivo anticancer efficacy}

The in vivo PDT efficacy of the conjugate RGD-(Linker) ${ }_{2}$-Glu-SiPc 7 was investigated using the U87-MG subcutaneous xenograft mouse model. Tumors were established in 8-week-old female BALB/c nude mice by subcutaneous inoculation of U87-MG cell suspension $\left(3 \times 10^{6}\right.$ cells per mouse) into the right thigh of each mouse. When the tumors reached $100 \mathrm{~mm}^{3}$ in volume, the mice were randomly divided into two groups (5 mice per group) with equivalent average starting tumor sizes. The treatment group was intravenously administered RGD-(Linker) ${ }_{2}$-Glu-SiPc 7 (50 nmol per mouse), followed by light illumination $\left(200 \mathrm{~mW} / \mathrm{cm}^{2}, 670 \mathrm{~nm}\right.$, $16 \mathrm{~min}) 4 \mathrm{~h}$ after the injection. The control group received an equivalent volume of PBS, followed by the same light illumination. Tumor volume and body weight evolution were monitored for all mice for 35 days after the PDT. Tumor volumes were measured with a digital caliper every other day and were calculated as follows: (length $\times$ width ${ }^{2}$ ) / 2 . Photographs of the mice were also taken every two days to observe the more visible tumor changes. Mice were considered dead when the tumor volume exceeded $1500 \mathrm{~mm}^{3}$.

\section{Supplementary Material}

Supplementary figures.

http://www.jcancer.org/v09p0310s1.pdf

\section{Acknowledgments}

This research was supported by the National Natural Science Foundation of China (NO. 31270926), the Ph.D. Candidate Research Innovation Fund of Nankai University (No. 96172128), the Major Program 
of the National Natural Science Foundation of China (No. 31527801) and the Natural Science Foundation of Tianjin (17JCYBJC24600).

\section{Note}

Electronic Supplementary Information (ESI) available: [Synthetic procedure for cRGDfK peptide and linker-modified peptides and HPLC chromatograms and ESI-HRMS of the conjugates]. See DOI:

\section{Competing Interests}

The authors have declared that no competing interest exists.

\section{References}

1. Agostinis P, Berg K, Cengel KA, et al. Photodynamic therapy of cancer: an update. CA Cancer J Clin. 2011; 61: 250-281.

2. Castano AP, Mroz P and Hamblin MR. Photodynamic therapy and anti-tumour immunity. Nat Rev Cancer. 2006; 6: 535.

3. Celli JP, Spring BQ, Rizvi I, et al. Imaging and photodynamic therapy: mechanisms, monitoring, and optimization. Chem Rev .2010; 110: 2795-2838.

4. Mitsunaga M, Ogawa M, Kosaka N, et al. Cancer cell-selective in vivo near infrared photoimmunotherapy targeting specific membrane molecules. Nat Med. 2011; 17: 1685-1691.

5. Ethirajan $\mathrm{M}$, Chen $\mathrm{Y}$, Joshi $\mathrm{P}$, et al. The role of porphyrin chemistry in tumor imaging and photodynamic therapy. Chem Soc Rev. 2011; 40: 340-362.

6. Jiang Z, Shao J, Yang T, et al. Pharmaceutical development, composition and quantitative analysis of phthalocyanine as the photosensitizer for cancer photodynamic therapy. J Pharmaceut Biomed. 2014; 87: 98-104.

7. Josefsen LB, Boyle RW. Unique diagnostic and therapeutic roles of porphyrins and phthalocyanines in photodynamic therapy, imaging and theranostics. Theranostics. 2012; 2: 916-966.

8. O'Connor AE, Gallagher WM, Byrne AT. Porphyrin and nonporphyrin photosensitizers in oncology: preclinical and clinical advances in photodynamic therapy. Photochem Photobiol. 2009; 85: 1053-1074.

9. Mckeown NB. Phthalocyanine materials: synthesis, structure and function. Cambridge University Press: UK. 1998; ISBN 0-521-49623-3.

10. Makhseed S, Machacek M, Alfadly W, et al. Water-soluble non-aggregating zinc phthalocyanine and in vitro studies for photodynamic therapy. Chem Commun. 2013; 49: 11149-11151.

11. DeRosa MC, Crutchley RJ. Photosensitized singlet oxygen and its applications. Chem Rev. 2002; 233: 351-371.

12. Lunardi CN, Tedesco AC. Synergic photosensitizers: a new trend in photodynamic therapy. Curr Org Chem. 2005; 9: 813-821.

13. Bugaj AM. Targeted photodynamic therapy-a promising strategy of tumor treatment. Photoch Photobio Sci. 2011; 10: 1097-1109.

14. Luan L, Fang W, Liu W, et al. Phthalocyanine-cRGD conjugate: synthesis, photophysical properties and in vitro biological activity for targeting photodynamic therapy. Org Biomol Chem. 2016; 14: 2985-2992.

15. Mahato R, Tai W, Cheng K. Prodrugs for improving tumor targetability and efficiency. Adv Drug Deliver Rev. 2011; 63: 659-670.

16. Ranyuk E, Cauchon N, Klarskov K, et al. Phthalocyanine-peptide conjugates: receptor-targeting bifunctional agents for imaging and photodynamic therapy. J Med Chem. 2013; 56: 1520-1534.

17. Ongarora BG, Fontenot $\mathrm{KR}, \mathrm{Hu} \mathrm{X}$, et al. Phthalocyanine-peptide conjugates for epidermal growth factor receptor targeting. J Med Chem. 2012; 55: 3725-3738.

18. Li YX, Wang J, Zhang XX, et al. Highly water-soluble and tumor-targeted photosensitizers for photodynamic therapy. Org Biomol Chem. 2015; 13: 7681-7694

19. Li F, Liu Q, Liang ZZ, et al. Synthesis and biological evaluation of peptideconjugated phthalocyanine photosensitizers with highly hydrophilic modifications. Org Biomol Chem. 2016; 14: 3409-3422.

20. Xu P, Chen J, Chen Z, et al. Receptor-targeting phthalocyanine photosensitizer for improving antitumor photocytotoxicity. PloS ONE. 2012; 7: e37051.

21. Sibrian-Vazquez M, Ortiz J, Nesterova IV, et al. Synthesis and properties of cell-targeted $\mathrm{Zn}(\mathrm{II})$-phthalocyanine-peptide conjugates. Bioconjugate Chem. 2007; 18 : 410-420.

22. Kucinska M, Skupin-Mrugalska P, Szczolko W, et al. Phthalocyanine derivatives possessing 2-(morpholin-4-yl)ethoxy groups as potential agents for photodynamic therapy. J Med Chem. 2015; 58: 2240-2255.

23. Machacek M, Cidlina A, Novakova V, et al. Far-red-absorbing cationic phthalocyanine photosensitizers: synthesis and evaluation of the photodynamic anticancer activity and the mode of cell death induction. J Med Chem. 2015; 58: 1736-1749.
24. Lo PC, Chan CMH, Liu JY, et al. Highly photocytotoxic glucosylated silicon(IV) phthalocyanines. Effects of peripheral chloro substitution on the photophysical and photodynamic properties. J Med Chem. 2007; 50: 2100-2107.

25. Lau JTF, Lo PC, Tsang YM, et al. Unsymmetrical $\beta$-cyclodextrin-conjugated silicon(IV) phthalocyanines as highly potent photosensitisers for photodynamic therapy. Chem Commun. 2011; 47: 9657-9659.

26. Hofman JW, Zeeland FV, Turker S, et al. Peripheral and axial substitution of phthalocyanines with solketal groups: synthesis and in vitro evaluation for photodynamic therapy. J Med Chem. 2007; 50: 1485-1494.

27. Jiang XJ, Lo PC, Tsang YM, et al. Phthalocyanine-polyamine conjugates as pH-controlled photosensitizers for photodynamic therapy. Chem Eur J. 2010; 16: 4777-4783.

28. Baron ED, Malbasa CL, Santo-Domingo D, et al. Silicon phthalocyanine (Pc 4) photodynamic therapy is a safe modality for cutaneous neoplasms: results of a phase 1 clinical trial. Laser Surg Med. 2010; 42: 728-735.

29. Miller JD, Baron ED, Scull H, et al. Photodynamic therapy with the phthalocyanine photosensitizer Pc 4: the case experience with preclinical mechanistic and early clinical-translational studies. Toxicol Appl Pharm. 2007; 224: 290-299.

30. Nkepang G, Bio M, Rajaputra P, et al. Folate receptor-mediated enhanced and specific delivery of far-red light-activatable prodrugs of combretastatin A-4 to FR-positive tumor. Bioconjugate Chem. 2014; 25: 2175-2188.

31. Thapa P, Li M, Bio M, et al. Far-red light-activatable prodrug of paclitaxel for the combined effects of photodynamic therapy and site-specific paclitaxel chemotherapy. J Med Chem. 2016; 59: 3204-3214.

32. Wang F, Li Y, Shen Y, et al. The functions and applications of RGD in tumor therapy and tissue engineering. Int J Mol Sci. 2013; 14: 13447-13462.

33. Chen $K$, Chen $X$. Integrin targeted delivery of chemotherapeutics. Theranostics. 2011; 1: 189-200.

34. Ke MR, Ng DKP, Lo PC. Synthesis and in vitro photodynamic activities of an integrin-targeting cRGD-conjugated Zinc(II) phthalocyanine. Chem Asian J. 2014; 9: 554-561

35. Guo Y, Yuan H, Rice WL, et al. The PEG-fluorochrome shielding approach for targeted probe design. J Am Chem Soc. 2012; 134: 19338-19341.

36. Liu F, Deng D, Chen $X$, et al. Folate-polyethylene glycol conjugated near-infrared fluorescence probe with high targeting affinity and sensitivity for in vivo early tumor diagnosis. Mol Imaging Biol. 2010; 12: 595-607.

37. Avinash S, Manivannan E, Suresh KP, et al. Conjugation of cRGD peptide to chlorophyll a based photosensitizer (HPPH) alters its pharmacokinetics with enhanced tumor-imaging and photosensitizing (PDT) efficacy. Mol Pharm. 2011; 8: 1186-1197. 